PROCEEDINGS OF THE

AMERICAN MATHEMATICAL SOCIETY

Volume 129, Number 2, Pages 415-427

S 0002-9939(00)05567-2

Article electronically published on August 28, 2000

\title{
CONVERGENCE OF CASCADE ALGORITHMS ASSOCIATED WITH NONHOMOGENEOUS REFINEMENT EQUATIONS
}

\author{
RONG-QING JIA, QINGTANG JIANG, AND ZUOWEI SHEN
}

(Communicated by David R. Larson)

\begin{abstract}
This paper is devoted to a study of multivariate nonhomogeneous refinement equations of the form

$$
\phi(x)=g(x)+\sum_{\alpha \in \mathbb{Z}^{s}} a(\alpha) \phi(M x-\alpha), \quad x \in \mathbb{R}^{s},
$$

where $\phi=\left(\phi_{1}, \ldots, \phi_{r}\right)^{T}$ is the unknown, $g=\left(g_{1}, \ldots, g_{r}\right)^{T}$ is a given vector of functions on $\mathbb{R}^{s}, M$ is an $s \times s$ dilation matrix, and $a$ is a finitely supported refinement mask such that each $a(\alpha)$ is an $r \times r$ (complex) matrix. Let $\phi_{0}$ be an initial vector in $\left(L_{2}\left(\mathbb{R}^{s}\right)\right)^{r}$. The corresponding cascade algorithm is given by

$$
\phi_{k}:=g+\sum_{\alpha \in \mathbb{Z}^{s}} a(\alpha) \phi_{k-1}(M \cdot-\alpha), \quad k=1,2, \ldots
$$

In this paper we give a complete characterization for the $L_{2}$-convergence of the cascade algorithm in terms of the refinement mask $a$, the nonhomogeneous term $g$, and the initial vector of functions $\phi_{0}$.
\end{abstract}

\section{INTRODUCTION}

A nonhomogeneous refinement equation is a functional equation of the form

$$
\phi(x)=g(x)+\sum_{\alpha \in \mathbb{Z}^{s}} a(\alpha) \phi(M x-\alpha), \quad x \in \mathbb{R}^{s},
$$

where $\phi=\left(\phi_{1}, \ldots, \phi_{r}\right)^{T}$ is the unknown, $g=\left(g_{1}, \ldots, g_{r}\right)^{T}$ is a given vector of functions on $\mathbb{R}^{s}, M$ is an $s \times s$ dilation matrix, and $a$ is a finitely supported mask such that each $a(\alpha)$ is an $r \times r$ (complex) matrix. In this paper, by a dilation matrix we mean an integer matrix whose eigenvalues lie outside the closed unit disk. When $g=0$, (1.1) becomes the homogeneous refinement equation

$$
\phi(x)=\sum_{\alpha \in \mathbb{Z}^{s}} a(\alpha) \phi(M x-\alpha), \quad x \in \mathbb{R}^{s} .
$$

Received by the editors June 29, 1998 and, in revised form, April 13, 1999.

2000 Mathematics Subject Classification. Primary 41A58, 42C40; Secondary 41A17, 42C99.

Key words and phrases. Nonhomogeneous refinement equations, cascade algorithms.

The first author was supported in part by NSERC Canada under Grant OGP 121336, and the second and third authors were supported in part by the Wavelets Strategic Research Programme, National University of Singapore. 
The readers are referred to [1] and [4 for some basic properties of homogeneous refinement equations. For vector homogeneous refinement equations, see [9], [2], [22], 19], and [27.

Nonhomogeneous refinement equations generalized from their homogeneous counterpart are motivated by constructions of multiwavelets to obtain multi-channel filters with good time-frequency localization in the area of signal processing and constructions of wavelets on a finite interval to find numerical solutions of differential equations in the area of numerical analysis (see, e.g., 24] and [3]). A systematic study of such refinement equations not only complements the existing literature of its homogeneous counterpart, but also provides a global view of the subject. This further leads to a better understanding of refinement equations.

For a given equation (1.1), the first problem to consider is whether it has distributional solutions. Distributional solutions of the nonhomogeneous refinement equation (1.1) were studied in [5], 25], 6], 15], and [26]. In [15, we provided a characterization of the existence of distributional solutions for continuous and discrete nonhomogeneous refinement equations.

In practice, solutions are required to be functions with certain smoothness. Since solutions are not of any analytic form in many cases, the cascade algorithm is used to obtain approximations of the solutions. The second problem to consider is the $L_{2^{-}}$ convergence of the cascade algorithm, which will be the main topic of this paper. The convergence of the cascade algorithm is fundamental to wavelet theory and subdivision. For example, in the context of wavelet theory, the key step to the construction is to find right refinable functions, and in the context of subdivision, the limiting surface of the subdivision process is a linear combination of shifts of the refinable function corresponding to the subdivision scheme.

Suppose $g=\left(g_{1}, \ldots, g_{r}\right)^{T}$ is a given $r \times 1$ vector of compactly supported functions in $L_{2}\left(\mathbb{R}^{s}\right)$. Let $Q_{a}$ be the cascade operator on $\left(L_{2}\left(\mathbb{R}^{s}\right)\right)^{r}$ given by

$$
Q_{a} f:=\sum_{\alpha \in \mathbb{Z}^{s}} a(\alpha) f(M \cdot-\alpha), \quad f=\left(f_{1}, \ldots, f_{r}\right)^{T} \in\left(L_{2}\left(\mathbb{R}^{s}\right)\right)^{r} .
$$

Choose an initial vector $\phi_{0} \in\left(L_{2}\left(\mathbb{R}^{s}\right)\right)^{r}$. Let

$$
\phi_{k}:=g+\sum_{\alpha \in \mathbb{Z}^{s}} a(\alpha) \phi_{k-1}(M \cdot-\alpha), \quad k=1,2, \ldots
$$

If there exists an $r \times 1$ vector of functions $\phi \in\left(L_{2}\left(\mathbb{R}^{s}\right)\right)^{r}$ such that

$$
\lim _{k \rightarrow \infty}\left\|\phi_{k}-\phi\right\|_{\left(L_{2}\left(\mathbb{R}^{s}\right)\right)^{r}}=0,
$$

then we say that the cascade algorithm associated with $a, g$, and $\phi_{0}$ is $L_{2^{-}}$ convergent. If this is the case, then the limit $\phi$ is a solution of the nonhomogeneous refinement equation (1.1). In the scalar case $(r=1)$, we say that the cascade algorithm associated with the corresponding homogeneous equation converges if it converges for any initial function $\phi_{0}$ that satisfies

$$
\sum_{\alpha \in \mathbb{Z}^{s}} \phi_{0}(\cdot-\alpha)=1
$$

The $L_{2}$-convergence of cascade algorithms associated with homogeneous refinement equations were investigated in many papers such as [11], [23], [8], [20], [22] and [16]. As it has been done in many other areas of mathematics, to solve the nonhomogeneous problem, one starts with the corresponding homogeneous problem and uses the results of this case as indicators and the methods as a starting 
point. This process normally is nontrivial, since the nonhomogeneous problem cannot be reduced to the homogeneous problem entirely. This is also the case for the convergence of the cascade algorithm as shown in the following two examples.

The first example shows that in the nonhomogeneous case, the choice of the initial functions highly depends on the nonhomogeneous term.

Example 1.1. Let $M=(2), a(0)=a(1)=1, a(\alpha)=0$ for all $\alpha \in \mathbb{Z} \backslash\{0,1\}$, and the nonhomogeneous term $g=\chi_{[0,1 / 4)}+2 \chi_{[1 / 4,1 / 2)}+\chi_{[3 / 4,1)}-\chi_{[1,2)}$, where $\chi_{E}$ denotes the characteristic function of the set $E$. It is clear that the cascade algorithm associated with the homogeneous equation converges for any initial function satisfies (1.4). However, since $\sum_{\alpha \in \mathbb{Z}} g(\cdot-\alpha) \neq 0$, the cascade algorithm associated with the nonhomogeneous equation does not converge for any initial function satisfying (1.4) by Remark [2.7] It converges when $\phi_{0}=\chi_{[0,1 / 2)}$.

Assume that the cascade algorithm associated with the homogeneous case converges. Corollary 2.6 provides a complete characterization of the initial functions for which the cascade algorithm associated with the nonhomogeneous refinement equation converges. However, the next example shows that the convergence of the cascade algorithm associated with the homogeneous refinement equation is not a necessary condition for the convergence of the cascade algorithm associated with the nonhomogeneous one. Hence, it is necessary to give a complete characterization in terms of the refinement mask $a$, the nonhomogeneous term $g$ and the initial vector of functions $\phi_{0}$ for the convergence of the cascade algorithm in the nonhomogeneous case. Such a characterization will be given in Theorem 2.4

Example 1.2. Consider the nonhomogeneous refinement equation

$$
\phi=a(\phi(2 \cdot)+\phi(2 \cdot-1))+g,
$$

where $1<a<\sqrt{2}$ and $g=\chi_{[0,1)}-\chi_{[1,2)}$. Clearly, the cascade algorithm associated with the corresponding homogeneous refinement equation does not converge in $L_{2^{-}}$ norm. On the other hand, since $\sum_{\alpha \in \mathbb{Z}} g(\cdot-\alpha)=0$, the cascade algorithm associated with the nonhomogeneous refinement equation does converge for any initial function $\phi_{0}$ supported in $[0,2]$ that satisfies $\sum_{\alpha \in \mathbb{Z}} \phi_{0}(\cdot-\alpha)=0$ as shown in Example 3.2.

In the univariate and scalar case $(s=1$ and $r=1)$, a characterization of $L_{2}$ convergence was given in [25] for cascade algorithms associated with the nonhomogeneous refinement equation (1.1). Their argument is based on the fact established in 12 that a compactly supported function on $\mathbb{R}$ is a linear combination of finitely many shifts of a compactly supported function whose shifts are linearly independent. However, multivariate compactly supported functions do not have such property (see [13] for a counterexample). Thus, a new technique has to be introduced to deal with the multivariate case.

The purpose of this paper is to give a complete characterization for the $L_{2}$ convergence of cascade algorithms in terms of the refinement mask $a$, the nonhomogeneous term $g$, and the initial vector of functions $\phi_{0}$. This will be done in Section 2. In Section 2, we also discuss some relation between the $L_{2}$-convergences of cascade algorithms associated with the homogeneous and nonhomogeneous refinement equations. In Section 3, we give several examples to illustrate the general theory. 


\section{Convergence of CASCAde Algorithms}

In this section we give a characterization for the $L_{2}$-convergence of the cascade algorithm associated with a nonhomogeneous refinement equation.

As usual, we use $L_{2}\left(\mathbb{R}^{s}\right)$ to denote the space of square integrable functions on $\mathbb{R}^{s}$. The norm on $L_{2}\left(\mathbb{R}^{s}\right)$ is given by

$$
\|f\|_{2}:=\left(\int_{\mathbb{R}^{s}}|f(x)|^{2} d x\right)^{1 / 2}, \quad f \in L_{2}\left(\mathbb{R}^{s}\right) .
$$

Given a measurable function $f$ on $\mathbb{R}^{s}$, we use $\|f\|_{\infty}$ to denote the essential supremum of $|f|$ on $\mathbb{R}^{s}$.

For two functions $f, h$ in $L_{2}\left(\mathbb{R}^{s}\right), f \odot h$ is defined as follows:

$$
f \odot h(x):=\int_{\mathbb{R}^{s}} f(x+y) \overline{h(y)} d y, \quad x \in \mathbb{R}^{s},
$$

where $\overline{h(y)}$ denotes the complex conjugate of $h(y)$. In other words, $f \odot h$ is the convolution of $f$ with the function $y \mapsto \overline{h(-y)}, y \in \mathbb{R}^{s}$. We find that this notation, which was introduced in [14, is convenient. It is easily seen that $f \odot h$ lies in $C_{0}\left(\mathbb{R}^{s}\right)$, the space of continuous functions on $\mathbb{R}^{s}$ which vanish at $\infty$. In particular, $f \odot h$ is uniformly continuous. Clearly,

$$
\|f \odot h\|_{\infty} \leq\|f\|_{2}\|h\|_{2} .
$$

Moreover, $(f \odot f)(0)=\|f\|_{2}^{2}$.

Let $\ell_{0}\left(\mathbb{Z}^{s}\right)$ denote the linear space of all finitely supported sequences on $\mathbb{Z}^{s}$, and let $\ell_{\infty}\left(\mathbb{Z}^{s}\right)$ denote the linear space of all bounded sequences on $\mathbb{Z}^{s}$. The norm on $\ell_{\infty}\left(\mathbb{Z}^{s}\right)$ is given by $\|v\|_{\infty}:=\sup \left\{|v(\alpha)|: \alpha \in \mathbb{Z}^{s}\right\}, v \in \ell_{\infty}\left(\mathbb{Z}^{s}\right)$.

We use $\mathbb{C}^{r}$ to denote the linear space of all $r \times 1$ complex vectors. The norm of a vector $\xi=\left(\xi_{1}, \ldots, \xi_{r}\right)^{T} \in \mathbb{C}^{r}$ is defined by $|\xi|:=\sum_{j=1}^{r}\left|\xi_{j}\right|$. If $F$ is a linear space, we use $F^{r}$ to denote the linear space

$$
\left\{\left(f_{1}, \ldots, f_{r}\right)^{T}: f_{1}, \ldots, f_{r} \in F\right\} .
$$

If, in addition, $F$ is a Banach space equipped with the norm $\|\cdot\|$, then $F^{r}$ is also a Banach space with the norm given by

$$
\|f\|:=\sum_{j=1}^{r}\left\|f_{j}\right\|, \quad f=\left(f_{1}, \ldots, f_{r}\right)^{T} \in F^{r} .
$$

The Kronecker product of two matrices is a useful tool in the study of vector refinement equations (see [7], [17] and [18]). Let us recall some basic properties of the Kronecker product from [10]. Suppose $A=\left(a_{i j}\right)_{1 \leq i \leq m, 1 \leq j \leq n}$ and $B=$ $\left(b_{i j}\right)_{1 \leq i \leq k, 1 \leq j \leq l}$ are two matrices. The (right) Kronecker product of $A$ and $B$, written $A \otimes B$, is defined to be the block matrix

$$
A \otimes B:=\left[\begin{array}{cccc}
a_{11} B & a_{12} B & \cdots & a_{1 n} B \\
a_{21} B & a_{22} B & \cdots & a_{2 n} B \\
\vdots & \vdots & \ddots & \vdots \\
a_{m 1} B & a_{m 2} B & \cdots & a_{m n} B
\end{array}\right] .
$$

For three matrices $A, B$, and $C$ of the same type, we have

$$
\begin{aligned}
& (A+B) \otimes C=(A \otimes C)+(B \otimes C), \\
& A \otimes(B+C)=(A \otimes B)+(A \otimes C) .
\end{aligned}
$$


If $A, B, C, D$ are four matrices such that the products $A C$ and $B D$ are well defined, then

$$
(A \otimes B)(C \otimes D)=(A C) \otimes(B D) .
$$

Moreover, if $\lambda_{1}, \ldots, \lambda_{r}$ are the eigenvalues of an $r \times r$ matrix $A$ and $\mu_{1}, \ldots, \mu_{r}$ are the eigenvalues of an $r \times r$ matrix $B$, then the eigenvalues of $A \otimes B$ are $\lambda_{j} \mu_{k}$, $j, k=1, \ldots, r$.

The vector $\left(a_{11}, \ldots, a_{r 1}, a_{12}, \ldots, a_{r 2}, \ldots, a_{1 r}, \ldots, a_{r r}\right)^{T}$ is said to be the vecfunction of $A$ and written as $\operatorname{vec} A$ for a matrix $A=\left(a_{i j}\right)_{1 \leq i, j \leq r}$. Suppose $A, X$, and $B$ are three $r \times r$ matrices. Then we have (see [10])

$$
\operatorname{vec}(A X B)=\left(B^{T} \otimes A\right) \operatorname{vec} X .
$$

Suppose $\phi=\left(\phi_{1}, \ldots, \phi_{r}\right)^{T}$ and $\psi=\left(\psi_{1}, \ldots, \psi_{r}\right)^{T}$ lie in $\left(L_{2}\left(\mathbb{R}^{s}\right)\right)^{r}$. Let $\phi \odot \psi^{T}$ be defined as follows:

$$
\phi \odot \psi^{T}:=\left[\begin{array}{cccc}
\phi_{1} \odot \psi_{1} & \phi_{1} \odot \psi_{2} & \cdots & \phi_{1} \odot \psi_{r} \\
\phi_{2} \odot \psi_{1} & \phi_{2} \odot \psi_{2} & \cdots & \phi_{2} \odot \psi_{r} \\
\vdots & \vdots & \ddots & \vdots \\
\phi_{r} \odot \psi_{1} & \phi_{r} \odot \psi_{2} & \cdots & \phi_{r} \odot \psi_{r}
\end{array}\right] .
$$

By (2.1) we have

$$
\left\|\operatorname{vec}\left(\phi \odot \psi^{T}\right)\right\|_{\infty} \leq\|\phi\|_{2}\|\psi\|_{2} .
$$

Moreover,

$$
\left|\operatorname{vec}\left(\phi \odot \phi^{T}\right)(0)\right|=\sum_{j=1}^{r} \sum_{k=1}^{r}\left|\phi_{j} \odot \phi_{k}(0)\right| \geq \sum_{j=1}^{r}\left|\phi_{j} \odot \phi_{j}(0)\right|=\sum_{j=1}^{r}\left\|\phi_{j}\right\|_{2}^{2} .
$$

Consequently,

$$
\left|\operatorname{vec}\left(\phi \odot \phi^{T}\right)(0)\right| \geq \frac{1}{r}\|\phi\|_{2}^{2} .
$$

Now let us discuss properties of the cascade operator defined in (1.2).

Lemma 2.1. Let $a_{k}(k=1,2, \ldots)$ be the sequences of $r \times r$ matrices defined by $a_{1}:=a$ and

$$
a_{k}(\alpha)=\sum_{\beta \in \mathbb{Z}^{s}} a_{k-1}(\beta) a(\alpha-M \beta), \quad \alpha \in \mathbb{Z}^{s}, k=2,3, \ldots
$$

Then the following formula is valid for all $k=1,2, \ldots$ and all $f \in\left(L_{2}\left(\mathbb{R}^{s}\right)\right)^{r}$ :

$$
Q_{a}^{k} f=\sum_{\alpha \in \mathbb{Z}^{s}} a_{k}(\alpha) f\left(M^{k} \cdot-\alpha\right)
$$

Proof. The proof proceeds by induction on $k$. For $k=1$, (2.5) comes from the definition of the cascade operator $Q_{a}$. Suppose $k>1$ and (2.5) has been verified 
for $k-1$. Then by the induction hypothesis we have

$$
\begin{aligned}
Q_{a}^{k} f & =Q_{a}^{k-1}\left(Q_{a} f\right)=\sum_{\beta \in \mathbb{Z}^{s}} a_{k-1}(\beta)\left(Q_{a} f\right)\left(M^{k-1} \cdot-\beta\right) \\
& =\sum_{\beta \in \mathbb{Z}^{s}} \sum_{\alpha \in \mathbb{Z}^{s}} a_{k-1}(\beta) a(\alpha) f\left(M^{k} \cdot-M \beta-\alpha\right) \\
& =\sum_{\alpha \in \mathbb{Z}^{s}}\left[\sum_{\beta \in \mathbb{Z}^{s}} a_{k-1}(\beta) a(\alpha-M \beta)\right] f\left(M^{k} \cdot-\alpha\right) \\
& =\sum_{\alpha \in \mathbb{Z}^{s}} a_{k}(\alpha) f\left(M^{k} \cdot-\alpha\right) .
\end{aligned}
$$

This completes the induction procedure.

Lemma 2.2. The following relation is valid for all $k=1,2, \ldots$ and all $f \in$ $\left(L_{2}\left(\mathbb{R}^{s}\right)\right)^{r}$ :

$$
\operatorname{vec}\left(\left(Q_{a}^{k} f\right) \odot\left(Q_{a}^{k} f\right)^{T}\right)=Q_{b}^{k}\left(\operatorname{vec}\left(f \odot f^{T}\right)\right),
$$

where $b$ is given by

$$
b(\alpha)=\sum_{\beta \in \mathbb{Z}^{s}} \overline{a(\beta)} \otimes a(\alpha+\beta) /|\operatorname{det} M|, \quad \alpha \in \mathbb{Z}^{s} .
$$

Proof. Write $m$ for $|\operatorname{det} M|$. It is easily seen that

$$
f\left(M^{k} \cdot-\alpha\right) \odot f^{T}\left(M^{k} \cdot-\beta\right)=\frac{1}{m^{k}}\left(f \odot f^{T}\right)\left(M^{k} \cdot-\alpha+\beta\right), \quad \alpha, \beta \in \mathbb{Z}^{s} .
$$

By (2.5) it follows that

$$
\begin{aligned}
\left(Q_{a}^{k} f\right) \odot\left(Q_{a}^{k} f\right)^{T} & =\left[\sum_{\alpha \in \mathbb{Z}^{s}} a_{k}(\alpha) f\left(M^{k} \cdot-\alpha\right)\right] \odot\left[\sum_{\beta \in \mathbb{Z}^{s}} a_{k}(\beta) f\left(M^{k} \cdot-\beta\right)\right]^{T} \\
& =\frac{1}{m^{k}} \sum_{\alpha \in \mathbb{Z}^{s}} \sum_{\beta \in \mathbb{Z}^{s}} a_{k}(\alpha)\left(f \odot f^{T}\right)\left(M^{k} \cdot-\alpha+\beta\right){\overline{a_{k}(\beta)}}^{T} .
\end{aligned}
$$

Therefore, with $h:=\operatorname{vec}\left(f \odot f^{T}\right)$, by (2.2) we obtain

$$
\begin{aligned}
\operatorname{vec}\left(\left(Q_{a}^{k} f\right) \odot\left(Q_{a}^{k} f\right)^{T}\right) & =\frac{1}{m^{k}} \sum_{\alpha \in \mathbb{Z}^{s}} \sum_{\beta \in \mathbb{Z}^{s}} \overline{a_{k}(\beta)} \otimes a_{k}(\alpha) h\left(M^{k} \cdot-\alpha+\beta\right) \\
& =\frac{1}{m^{k}} \sum_{\alpha \in \mathbb{Z}^{s}} \sum_{\beta \in \mathbb{Z}^{s}} \overline{a_{k}(\beta)} \otimes a_{k}(\alpha+\beta) h\left(M^{k} \cdot-\alpha\right) .
\end{aligned}
$$

Let $b_{k}(k=1,2, \ldots)$ be the sequences of $r^{2} \times r^{2}$ matrices defined by $b_{1}:=b$ and

$$
b_{k}(\alpha)=\sum_{\beta \in \mathbb{Z}^{s}} b_{k-1}(\beta) b(\alpha-M \beta), \quad \alpha \in \mathbb{Z}^{s}, k=2,3, \ldots
$$

Then one can show as in the proof of (2.5) that

$$
Q_{b}^{k} h=\sum_{\alpha \in \mathbb{Z}^{s}} b_{k}(\alpha) h\left(M^{k} \cdot-\alpha\right) .
$$

To complete the proof of Lemma 2.2, it suffices to show

$$
b_{k}(\alpha)=\frac{1}{m^{k}} \sum_{\beta \in \mathbb{Z}^{s}} \overline{a_{k}(\beta)} \otimes a_{k}(\alpha+\beta) \quad \forall \alpha \in \mathbb{Z}^{s} .
$$


This will be done by induction on $k$. By the definitions of $a_{1}$ and $b_{1}$, (2.7) is true for $k=1$. Suppose $k>1$ and (2.7) has been verified for $k-1$. By the induction hypothesis, for $\alpha \in \mathbb{Z}^{s}$ we have

$$
\begin{aligned}
b_{k}(\alpha) & =\sum_{\eta \in \mathbb{Z}^{s}} b_{k-1}(\eta) b(\alpha-M \eta) \\
& =m^{-k} \sum_{\beta \in \mathbb{Z}^{s}} \sum_{\gamma \in \mathbb{Z}^{s}} \sum_{\eta \in \mathbb{Z}^{s}}\left(\overline{a_{k-1}(\gamma)} \otimes a_{k-1}(\eta+\gamma)\right)(\overline{a(\beta)} \otimes a(\alpha-M \eta+\beta)) \\
& =m^{-k} \sum_{\beta \in \mathbb{Z}^{s}} \sum_{\gamma \in \mathbb{Z}^{s}} \sum_{\eta \in \mathbb{Z}^{s}}\left(\overline{a_{k-1}(\gamma) a(\beta-M \gamma)}\right) \otimes\left(a_{k-1}(\eta) a(\alpha+\beta-M \eta)\right) \\
& =m^{-k} \sum_{\beta \in \mathbb{Z}^{s}} \overline{a_{k}(\beta)} \otimes a_{k}(\alpha+\beta) .
\end{aligned}
$$

This completes the induction procedure, and thereby finishes the proof of Lemma 2.2 .

Let $T_{b}$ be the transition operator on $\left(\ell_{0}\left(\mathbb{Z}^{s}\right)\right)^{r^{2}}$ given by

$$
T_{b} v(\alpha):=\sum_{\beta \in \mathbb{Z}^{s}} b(M \alpha-\beta) v(\beta), \quad \alpha \in \mathbb{Z}^{s}, v \in\left(\ell_{0}\left(\mathbb{Z}^{s}\right)\right)^{r^{2}} .
$$

It is known that the minimal invariant subspace $V$ of $T_{b}$ generated by $v$ is finite dimensional (see [8] and [16]). We use $\rho\left(\left.T_{b}\right|_{V}\right)$ to denote the spectral radius of $\left.T_{b}\right|_{V}$.

Lemma 2.3. The following relation is valid for all $k=1,2, \ldots$ and all $v \in$ $\left(\ell_{0}\left(\mathbb{Z}^{s}\right)\right)^{r^{2}}$ :

$$
T_{b}^{k} v(\alpha)=\sum_{\beta \in \mathbb{Z}^{s}} b_{k}\left(M^{k} \alpha-\beta\right) v(\beta), \quad \alpha \in \mathbb{Z}^{s}, k=1,2, \ldots
$$

Proof. The proof proceeds by induction on $k$. By the definition of $T_{b}$, our claim is true for $k=1$. Suppose it is valid for $k-1$. By the induction hypothesis, for $\alpha \in \mathbb{Z}^{s}$ we have

$$
\begin{aligned}
T_{b}^{k} v(\alpha) & =T_{b}^{k-1}\left(T_{b} v\right)(\alpha) \\
& =\sum_{\beta \in \mathbb{Z}^{s}} b_{k-1}\left(M^{k-1} \alpha-\beta\right)\left(T_{b} v\right)(\beta) \\
& =\sum_{\beta \in \mathbb{Z}^{s}} \sum_{\gamma \in \mathbb{Z}^{s}} b_{k-1}\left(M^{k-1} \alpha-\beta\right) b(M \beta-\gamma) v(\gamma) \\
& =\sum_{\gamma \in \mathbb{Z}^{s}}\left[\sum_{\beta \in \mathbb{Z}^{s}} b_{k-1}(\beta) b\left(M^{k} \alpha-\gamma-M \beta\right)\right] v(\gamma) \\
& =\sum_{\gamma \in \mathbb{Z}^{s}} b_{k}\left(M^{k} \alpha-\gamma\right) v(\gamma) .
\end{aligned}
$$

The proof of Lemma 2.3 is complete.

Let us investigate the cascade algorithm as given in (1.3). For $k=1,2, \ldots$, by (1.2) and (1.3) we have $\phi_{k}=g+Q_{a} g+\cdots+Q_{a}^{k-1} g+Q_{a}^{k} \phi_{0}$. It follows that

$$
\phi_{k+1}-\phi_{k}=Q_{a}^{k} g+Q_{a}^{k+1} \phi_{0}-Q_{a}^{k} \phi_{0}=Q_{a}^{k} g_{0},
$$

where $g_{0}:=g+Q_{a} \phi_{0}-\phi_{0}$. The following theorem gives a characterization for the $L_{2}$-convergence of the cascade algorithm associated with (1.1). 
Theorem 2.4. The cascade algorithm associated with $a, g$, and $\phi_{0}$ converges in the $L_{2}$-norm if and only if $\lim _{k \rightarrow \infty}\left\|T_{b}^{k} v\right\|_{\infty}=0$, where $b$ is given by (2.6) and $v$ is given by $v(\alpha)=\operatorname{vec}\left(g_{0} \odot g_{0}^{T}\right)(\alpha), g_{0}=g+Q_{a} \phi_{0}-\phi_{0}, \alpha \in \mathbb{Z}^{s}$, or equivalently, $\rho\left(\left.T_{b}\right|_{V}\right)<1$, where $V$ is the minimal invariant subspace of $T_{b}$ generated by $v$.

Proof. Let us first establish the sufficiency part of the theorem. Write $h_{0}$ for $\operatorname{vec}\left(g_{0} \odot g_{0}^{T}\right)$ and $h_{k}$ for $\operatorname{vec}\left(\left(Q_{a}^{k} g_{0}\right) \odot\left(Q_{a}^{k} g_{0}\right)^{T}\right), k=1,2, \ldots$ Then $v(\alpha)=h_{0}(\alpha)$ for all $\alpha \in \mathbb{Z}^{s}$. By Lemma 2.2 we have $h_{k}=Q_{b}^{k} h_{0}, k=1,2, \ldots$ An application of (2.4) gives

$$
\left\|Q_{a}^{k} g_{0}\right\|_{2}^{2} \leq r\left|\operatorname{vec}\left(\left(Q_{a}^{k} g_{0}\right) \odot\left(Q_{a}^{k} g_{0}\right)^{T}\right)(0)\right|=r\left|h_{k}(0)\right| .
$$

For $\alpha \in \mathbb{Z}^{s}$, by Lemma 2.3 and Lemma 2.2 we have

$$
T_{b}^{k} v(\alpha)=\sum_{\beta \in \mathbb{Z}^{s}} b_{k}\left(M^{k} \alpha-\beta\right) v(\beta)=\sum_{\beta \in \mathbb{Z}^{s}} b_{k}(\beta) h_{0}\left(M^{k} \alpha-\beta\right)=Q_{b}^{k} h_{0}(\alpha) .
$$

In particular, $h_{k}(0)=Q_{b}^{k} h_{0}(0)=T_{b}^{k} v(0)$. If $\rho\left(\left.T_{b}\right|_{V}\right)<1$, then there exists some $\eta, 0<\eta<1$, and a constant $C>0$ such that $\left|T_{b}^{k} v(0)\right| \leq\left\|T_{b}^{k} v\right\|_{\infty} \leq C \eta^{k}$. Consequently,

$$
\left\|Q_{a}^{k} g_{0}\right\|_{2}^{2} \leq r\left|h_{k}(0)\right|=r\left|Q_{b}^{k} h_{0}(0)\right|=r\left|T_{b}^{k} v(0)\right| \leq C r \eta^{k}, \quad k=1,2, \ldots .
$$

In light of (2.8), we have $\phi_{k+1}-\phi_{k}=Q_{a}^{k} g_{0}$. Since $\eta<1,\left(\phi_{k}\right)_{k=1,2, \ldots}$ is a Cauchy sequence in $\left(L_{2}\left(\mathbb{R}^{s}\right)\right)^{r}$. Therefore, the cascade algorithm converges in the $L_{2}$-norm.

Next, we establish the necessity part of the theorem. By Lemma 2.2 and (2.3) we obtain

$$
\left|Q_{b}^{k} h_{0}(\alpha)\right|=\left|\operatorname{vec}\left(\left(Q_{a}^{k} g_{0}\right) \odot\left(Q_{a}^{k} g_{0}\right)^{T}\right)(\alpha)\right| \leq\left\|Q_{a}^{k} g_{0}\right\|_{2}^{2} \quad \forall \alpha \in \mathbb{Z}^{s} .
$$

This in connection with (2.9) gives $\left\|T_{b}^{k} v\right\|_{\infty} \leq\left\|Q_{a}^{k} g_{0}\right\|_{2}^{2}$. If the cascade algorithm converges in the $L_{2}$-norm, then $\lim _{k \rightarrow \infty}\left\|Q_{a}^{k} g_{0}\right\|_{2}^{2}=0$. Hence, $\lim _{k \rightarrow \infty}\left\|T_{b}^{k} v\right\|_{\infty}=0$. Consequently, $\rho\left(\left.T_{b}\right|_{V}\right)<1$.

Remark 2.5. Let $K$ be a compact subset of $\mathbb{R}^{s}$ containing $\operatorname{supp} b:=\left\{\alpha \in \mathbb{Z}^{s}\right.$ : $b(\alpha) \neq 0\}$, and let $\Omega:=\left(\sum_{n=1}^{\infty} M^{-n} K\right) \cap \mathbb{Z}^{s}$. Choose $K$ properly so that $\Omega$ contains the support of $v$, where $v$ is given by $v(\alpha)=\operatorname{vec}\left(g_{0} \odot g_{0}^{T}\right)(\alpha), \alpha \in \mathbb{Z}^{s}$. Let $\mathcal{T}_{b}$ be the matrix $(b(M \alpha-\beta))_{\alpha, \beta \in \Omega}$. If $\rho\left(\mathcal{T}_{b}\right)<1$, then $\lim _{k \rightarrow \infty}\left\|T_{b}^{k} v\right\|_{\infty}=0$. Hence, the cascade algorithm associated with $a, g$, and any initial choice of $\phi_{0}$ is $L_{2}$-convergent. Suppose $\rho\left(\mathcal{T}_{b}\right) \geq 1$. Let $U$ be a nonsingular matrix satisfying $\mathcal{T}_{b}=$ $U^{-1} \operatorname{diag}\left(A_{1}, A_{2}\right) U$, where $A_{1}$ and $A_{2}$ are two square matrices such that $\rho\left(A_{1}\right)<1$ and the eigenvalues of $A_{2}$ lie outside the open unit disk. Suppose $A_{2}$ is an $m_{2} \times m_{2}$ matrix. Then $\lim _{k \rightarrow \infty}\left\|T_{b}^{k} v\right\|_{\infty}=0$ if and only if

$$
U_{2}(v(\beta))_{\beta \in \Omega}=0,
$$

where $U_{2}$ is the matrix consisting of the last $m_{2}$ rows of $U$. Therefore, the cascade algorithm associated with $a, g$, and $\phi_{0}$ is $L_{2}$-convergent if and only if (2.10) is true.

Next we will show that for a special set of vectors of compactly supported functions $g \in\left(L_{2}\left(\mathbb{R}^{s}\right)\right)^{r}$ the corresponding cascade algorithm converges as long as the cascade algorithm corresponding to the homogeneous refinement equation converges. 
For this purpose, consider the homogeneous refinement equation

$$
\varphi=\sum_{\alpha \in \mathbb{Z}^{s}} a(\alpha) \varphi(M \cdot-\alpha) .
$$

Assume that $|\operatorname{det} M|$ is a simple eigenvalue of the matrix $\sum_{\alpha \in \mathbb{Z}^{s}} a(\alpha)$ with a left row eigenvector $y$. We say that the cascade algorithm associated with the homogeneous refinement equation with mask $a$, converges (in the $L_{2}$-norm) whenever for any compactly supported $\varphi_{0} \in\left(L_{2}\left(\mathbb{R}^{s}\right)\right)^{r}$ satisfying

$$
y \sum_{\alpha \in \mathbb{Z}^{s}} \varphi_{0}(\cdot-\alpha)=y,
$$

the sequence $\varphi_{k}$ defined by $\varphi_{k}=\sum_{\alpha \in \mathbb{Z}^{s}} a(\alpha) \varphi_{k-1}(M \cdot-\alpha), k=1,2, \ldots$, converges in the $L_{2}$-norm.

Corollary 2.6. Assume that $|\operatorname{det} M|$ is a simple eigenvalue of the matrix $\sum_{\alpha \in \mathbb{Z}^{s}} a(\alpha)$ with a left row eigenvector $y$. Assume that the cascade algorithm associated with the homogeneous refinement equation with mask a converges in the $L_{2}$-norm. Then the cascade algorithm associated with $a, g$, and $\phi_{0}$ converges in the $L_{2}$-norm if and only if $g$ and $\phi_{0}$ satisfy

$$
y\left(\sum_{\alpha \in \mathbb{Z}^{s}}\left(g_{0} \odot g_{0}^{T}\right)(\alpha)\right) \bar{y}^{T}=0,
$$

where $g_{0}=g+Q_{a} \phi_{0}-\phi_{0}$.

Proof. If the cascade algorithm associated with the homogeneous refinement equation with mask $a$ converges in the $L_{2}$-norm, then 1 is a simple eigenvalue of $T_{b}$ and the other eigenvalues of $T_{b}$ lie inside the open unit disc (see [20 and 22]). Furthermore, the space $V$ defined by

$$
V:=\left\{u \in\left(\ell_{0}\left(\mathbb{Z}^{s}\right)\right)^{r^{2}}:(\bar{y} \otimes y) \sum_{\alpha \in \mathbb{Z}^{s}} u(\alpha)=0\right\}
$$

is invariant under $T_{b}$ and $\rho\left(\left.T_{b}\right|_{V}\right)<1$ (see [8] and [14]). Let $v$ be the element in $\left(\ell_{0}\left(\mathbb{Z}^{s}\right)\right)^{r^{2}}$ defined by $v(\alpha):=\operatorname{vec}\left(g_{0} \odot g_{0}^{T}\right)(\alpha), \alpha \in \mathbb{Z}^{s}$. In light of (2.2), it follows from (2.12) that $v$ lies in $V$. Hence, $\lim _{k \rightarrow \infty}\left\|T_{b}^{k} v\right\|_{\infty}=0$. By Theorem 2.4 the cascade algorithm associated with $a, g$, and $\phi_{0}$ converges in the $L_{2}$-norm.

Conversely, assume that the cascade algorithm associated with $a, g$ and $\phi_{0}$ converges. Let $w(\alpha):=\operatorname{vec}\left(\varphi \odot \varphi^{T}\right)(\alpha), \alpha \in \mathbb{Z}^{s}$, where $\varphi$ is the solution of the homogeneous refinement equation with mask $a$ such that $y \hat{\varphi}(0)=1$. Then $w$ is an eigenvector of $T_{b}$ corresponding to eigenvalue 1 with $(\bar{y} \otimes y) \sum_{\alpha \in \mathbb{Z}^{s}} w(\alpha)=1$.

Recall that $v$ is the element in $\left(\ell_{0}\left(\mathbb{Z}^{s}\right)\right)^{r^{2}}$ defined by $v(\alpha):=\operatorname{vec}\left(g_{0} \odot g_{0}^{T}\right)(\alpha), \alpha \in$ $\mathbb{Z}^{s}$. There exist $c \in \mathbb{C}$ and $u \in V$ such that $v=c w+u$. We have $\lim _{k \rightarrow \infty}\left\|T_{b}^{k} u\right\|_{\infty}=$ 0 . Since the cascade algorithm associated with $a, g$ and $\phi_{0}$ converges in the $L_{2^{-}}$ norm, we also have $\lim _{k \rightarrow \infty}\left\|T_{b}^{k} v\right\|_{\infty}=0$, by Theorem 2.4. However, $T_{b} w=w$. Hence,

$$
T_{b}^{k} v=c w+T_{b}^{k} u, \quad k=1,2, \ldots .
$$

Letting $k \rightarrow \infty$ in the above equation, we obtain $c=0$. Consequently, $v=u$ lies in $V$. In other words, (2.12) holds true.

Remark 2.7. In Corollary 2.6] condition (2.12) is equivalent to $y \sum_{\alpha \in \mathbb{Z}^{s}} g_{0}(\cdot-\alpha)=$ 0 . Assume that $y \sum_{\alpha \in \mathbb{Z}^{s}} \phi_{0}(\cdot-\alpha)=y$. Then, the cascade algorithm associated with nonhomogeneous equation converges if and only if $y \sum_{\alpha \in \mathbb{Z}^{s}} g(\cdot-\alpha)=0$. 


\section{EXAMPLES}

In this section we give several examples to illustrate our theory.

Example 3.1. Consider the nonhomogeneous refinement equation

$$
\phi(x)=t \phi(2 x)+g(x), \quad x \in \mathbb{R},
$$

where $t$ is a nonzero complex number and $g$ is a compactly supported function in $L_{2}(\mathbb{R})$. Let $\phi_{0}$ be a compactly supported function in $L_{2}(\mathbb{R})$. The corresponding cascade algorithm is given by $\phi_{k}=t \phi_{k-1}(2 \cdot)+g, k=1,2, \ldots$ We have $a(0)=t$ and $a(\alpha)=0$ for $\alpha \in \mathbb{Z} \backslash\{0\}$. Let $b$ be the sequence given by (2.6). Then $b(0)=|t|^{2} / 2$ and $b(\alpha)=0$ for $\alpha \in \mathbb{Z} \backslash\{0\}$. Let $g_{0}:=g+t \phi_{0}(2 \cdot)-\phi_{0}$ and $v(\alpha):=\left(g_{0} \odot g_{0}\right)(\alpha)$ for $\alpha \in \mathbb{Z}$. Then for sufficiently large $k$ we have

$$
T_{b}^{k} v(0)=\left(|t|^{2} / 2\right)^{k} v(0) \quad \text { and } \quad T_{b}^{k} v(\alpha)=0 \quad \forall \alpha \in \mathbb{Z} \backslash\{0\} .
$$

Note that $v(0)=\left\|g_{0}\right\|_{2}^{2}$. Hence, $v(0)=0$ if and only if $g_{0}=0$. If $|t|<\sqrt{2}$, then Theorem 2.4 tells us that the cascade algorithm associated with $a, g$, and any $\phi_{0}$ is $L_{2}$-convergent. If $|t| \geq \sqrt{2}$, then the cascade algorithm converges in the $L_{2}$-norm if and only if $\phi_{0}$ is the solution of the equation (3.1).

This example was also considered in 21 and 25$]$.

Example 3.2. Consider the nonhomogeneous refinement equation

$$
\phi(x)=a_{0} \phi(2 x)+a_{1} \phi(2 x-1)+g(x), \quad x \in \mathbb{R},
$$

where $a_{0}, a_{1}$ are two nonzero complex numbers and $g$ is a function in $L_{2}(\mathbb{R})$ supported in $[0,2]$. Let $\phi_{0}$ be a function in $L_{2}(\mathbb{R})$ supported in $[0,2]$. The corresponding cascade algorithm is given by

$$
\phi_{k}=a_{0} \phi_{k-1}(2 \cdot)+a_{1} \phi_{k-1}(2 \cdot-1)+g, \quad k=1,2, \ldots .
$$

We have $a(0)=a_{0}, a(1)=a_{1}$ and $a(\alpha)=0$ for $\alpha \in \mathbb{Z} \backslash\{0,1\}$. Let $b$ be the sequence given by (2.6). Then $b(-1)=a_{0} \bar{a}_{1} / 2, b(0)=\left(\left|a_{0}\right|^{2}+\left|a_{1}\right|^{2}\right) / 2, b(1)=\bar{a}_{0} a_{1} / 2$ and $b(\alpha)=0$ for $\alpha \in \mathbb{Z} \backslash\{-1,0,1\}$. Let $g_{0}:=g+a_{0} \phi_{0}(2 \cdot)+a_{1} \phi_{0}(2 \cdot-1)-\phi_{0}$ and $v(\alpha):=\left(g_{0} \odot g_{0}\right)(\alpha)$ for $\alpha \in \mathbb{Z}$. Then $g_{0}$ is supported in $[0,2]$ and $v(\alpha)=0$ for $\alpha \in \mathbb{Z} \backslash\{-1,0,1\}$.

Let $\mathcal{T}_{b}$ be the matrix $(b(2 i-j))_{-1 \leq i, j \leq 1}$; that is,

$$
\mathcal{T}_{b}=\left[\begin{array}{ccc}
b(-1) & 0 & 0 \\
b(1) & b(0) & b(-1) \\
0 & 0 & b(1)
\end{array}\right] .
$$

The eigenvalues of $\mathcal{T}_{b}$ are $a_{0} \bar{a}_{1} / 2, \bar{a}_{0} a_{1} / 2$ and $\left(\left|a_{0}\right|^{2}+\left|a_{1}\right|^{2}\right) / 2$. If $\left|a_{0}\right|^{2}+\left|a_{1}\right|^{2}<2$, then $\rho\left(\mathcal{T}_{b}\right)<1$. Hence the cascade algorithm associated with $\left\{a_{0}, a_{1}\right\}, g$ and $\phi_{0}$ is $L_{2}$-convergent. If $\left|a_{0} a_{1}\right| \geq 2$, then each eigenvalue of $\mathcal{T}_{b}$ lies outside the open unit disk. Therefore the cascade algorithm associated with $\left\{a_{0}, a_{1}\right\}, g$ and $\phi_{0}$ is $L_{2}$-convergent if and only if $\phi_{0}$ is the $L_{2}$-solution of (3.2).

Finally, we consider the case $\left|a_{0} a_{1}\right|<2 \leq\left|a_{0}\right|^{2}+\left|a_{1}\right|^{2}$. Let

$$
U:=\left[\begin{array}{ccc}
1 & 0 & 0 \\
0 & 0 & 1 \\
p & 1 & \bar{p}
\end{array}\right],
$$

where $p:=b(1) /(b(0)-b(-1))=\bar{a}_{0} a_{1} /\left(\left|a_{0}\right|^{2}+\left|a_{1}\right|^{2}-a_{0} \bar{a}_{1}\right)$. Then

$$
\mathcal{T}_{b}=U^{-1} \operatorname{diag}(b(-1), b(1), b(0)) U \text {. }
$$


By Theorem 2.4 and Remark 2.5 we conclude that the cascade algorithm associated with $\left\{a_{0}, a_{1}\right\}, g$ and $\phi_{0}$ is $L_{2}$-convergent if and only if $g$ and $\phi_{0}$ satisfy

$$
p\left(g_{0} \odot g_{0}\right)(-1)+\bar{p}\left(g_{0} \odot g_{0}\right)(1)+\left\|g_{0}\right\|_{2}^{2}=0 .
$$

Assume $a_{0}=a_{1}=a$ with $1 \leq|a|<\sqrt{2}$; then $p$ and $\bar{p}$ in (3.2) become 1. Thus (3.2) is equivalent to $\sum_{\alpha \in \mathbb{Z}} g_{0}(\cdot-\alpha)=0$. In particular, if $\sum_{\alpha \in \mathbb{Z}} g(\cdot-\alpha)=0$ and $\sum_{\alpha \in \mathbb{Z}} \phi_{0}(\cdot-\alpha)=0$ hold, then (3.2) is valid. Hence, the corresponding cascade algorithm converges.

Example 3.3. Consider the nonhomogeneous vector refinement equation

$$
\phi(x)=g(x)+\sum_{j=0}^{2} a(j) \phi(2 x-j), \quad x \in \mathbb{R},
$$

where $g=\left(g_{1}, g_{2}\right)^{T}$ is a $2 \times 1$ vector of compactly supported functions in $L_{2}(\mathbb{R})$, and

$$
a(0)=\left[\begin{array}{ll}
1 / 2 & 1 / 2 \\
1 / 4 & 1 / 4
\end{array}\right], \quad a(1)=\left[\begin{array}{cc}
1 & 0 \\
0 & 1 / 2
\end{array}\right], \quad a(2)=\left[\begin{array}{cc}
1 / 2 & -1 / 2 \\
-1 / 4 & 1 / 4
\end{array}\right] .
$$

Let $\phi_{0}=\left(\phi_{0,1}, \phi_{0,2}\right)^{T}$ be a $2 \times 1$ vector of compactly supported functions in $L_{2}(\mathbb{R})$. Then the cascade algorithm associated with $a, g$, and $\phi_{0}$ is $L_{2}$-convergent, provided

$$
\sum_{\alpha \in \mathbb{Z}} g_{1}(\cdot-\alpha)=0 \quad \text { and } \quad \sum_{\alpha \in \mathbb{Z}} \phi_{0,1}(\cdot-\alpha)=1
$$

In order to justify our claim, we first compute the sequence $b$ as given in (2.6). We have $b(\alpha)=0$ for $\alpha \in \mathbb{Z} \backslash[-2,2]$, and

$$
\begin{gathered}
b(-2)=[a(2) \otimes a(0)] / 2, \quad b(-1)=[a(1) \otimes a(0)+a(2) \otimes a(1)] / 2, \\
b(0)=[a(0) \otimes a(0)+a(1) \otimes a(1)+a(2) \otimes a(2)] / 2, \\
b(1)=[a(0) \otimes a(1)+a(1) \otimes a(2)] / 2, \quad b(2)=[a(0) \otimes a(2)] / 2 .
\end{gathered}
$$

Let $B$ be the block matrix $(b(2 \alpha-\beta))_{-2 \leq \alpha, \beta \leq 2}$. By a straightforward computation we find that 1 is a simple eigenvalue of the matrix $B$ and the other eigenvalues of $B$ are less than 1 in modulus. Note that 2 is a simple eigenvalue of the matrix $\sum_{\alpha \in \mathbb{Z}^{2}} a(\alpha)$ with $(1,0)$ being a left eigenvector. Further, one can check

$$
(1,0) \sum_{j=0}^{2} a(2 j+i)=(1,0), \quad i=0,1 .
$$

Thus the cascade algorithm associated with the homogeneous refinement equation with mask $a$ converges in the $L_{2}$-norm (see [22] and [16]). Hence, by Corollary 2.6 and Remark 2.7 the cascade algorithm associated with $a, g$, and $\phi_{0}$ converges provided the conditions in (3.5) are satisfied.

Example 3.4. Consider the nonhomogeneous vector refinement equation

$$
\phi(x)=A \phi(2 x)+g(x), \quad x \in \mathbb{R}^{2},
$$

where $A$ is a nonzero $2 \times 2$ matrix and $g=\left(g_{1}, g_{2}\right)^{T}$ is a $2 \times 1$ vector of compactly supported functions in $L_{2}\left(\mathbb{R}^{2}\right)$. Let $\phi_{0}=\left(\phi_{0,1}, \phi_{0,2}\right)^{T}$ be a $2 \times 1$ vector of compactly supported functions in $L_{2}\left(\mathbb{R}^{2}\right)$. The corresponding cascade algorithm is given by $\phi_{k}=A \phi_{k-1}(2 \cdot)+g, k=1,2, \ldots$ Let $g_{0}:=g+Q_{a} \phi_{0}-\phi_{0}$ and $v(\alpha):=\operatorname{vec}\left(g_{0} \odot g_{0}^{T}\right)(\alpha), \alpha \in \mathbb{Z}^{2}$. We observe that $v(0)=0$ if and only if $g_{0}=0$. 
In this case, $a(0)=A$ and $a(\alpha)=0$ for $\alpha \in \mathbb{Z}^{2} \backslash\{0\}$. Let $b$ be the sequence given in (2.6). Then

$$
b(0)=\bar{A} \otimes A / 4 \quad \text { and } \quad b(\alpha)=0 \quad \forall \alpha \in \mathbb{Z}^{2} \backslash\{0\} .
$$

Note that $\rho(\bar{A} \otimes A)=\rho(A)^{2}$. For sufficiently large $k$, we have

$$
T_{b}^{k} v(0)=(\bar{A} \otimes A / 4) v(0) \quad \text { and } \quad T_{b}^{k} v(\alpha)=0 \quad \forall \alpha \in \mathbb{Z}^{2} \backslash\{0\} .
$$

Thus, if $\rho(A)<2$, then $\rho\left(T_{b}\right)<1$ and the cascade algorithm associated with $A, g$, and any $\phi_{0}$ is $L_{2}$-convergent, by Theorem 2.4.

Let $\lambda_{1}, \lambda_{2}$ be the two eigenvalues of $A$. Then the eigenvalues of the matrix $\bar{A} \otimes A$ are $\left|\lambda_{1}\right|^{2}, \lambda_{1} \overline{\lambda_{2}}, \lambda_{2} \overline{\lambda_{1}}$, and $\left|\lambda_{2}\right|^{2}$. If $\left|\lambda_{1}\right| \geq 2$ and $\left|\lambda_{2}\right| \geq 2$, then the cascade algorithm associated with $A, g$, and $\phi_{0}$ is $L_{2}$-convergent only if $g_{0}=0$, i.e., $\phi_{0}$ is the solution of the refinement equation (3.6).

It remains to deal with the case $\left|\lambda_{2}\right|<2 \leq\left|\lambda_{1}\right|$. After a suitable coordinate change we may assume that $A$ has the following form $A=\operatorname{diag}\left(\lambda_{1}, \lambda_{2}\right)$. Then the cascade algorithm associated with $A, g$, and $\phi_{0}$ is $L_{2}$-convergent if and only if $\phi_{0,1}$ is the $L_{2}$-solution of the equation $\varphi=\lambda_{1} \varphi(2 \cdot)+g_{1}$.

\section{ACKNOWLEDGMENTS}

This research was supported in part by NSERC Canada under Grant OGP 121336 and the Wavelets Strategic Research Programme, National University of Singapore under a grant from the National Science and Technology Board and the Ministry of Education, Singapore.

\section{REFERENCES}

1. A. S. Cavaretta, W. Dahmen, and C. A. Micchelli, Stationary Subdivision, Memoirs of Amer. Math. Soc., vol. 93, 1991. MR 92h:65017

2. A. Cohen, I. Daubechies, and G. Plonka, Regularity of refinable function vectors, J. Fourier Anal. Appl. 3 (1997), 295-324. MR 98e:42031

3. A. Cohen, I. Daubechies, and P. Vial, Wavelets and fast wavelet transforms on an interval, Appl. Comp. Harm. Anal. 1 (1993), 54-81. MR 94m:42074

4. I. Daubechies and J. C. Lagarias, Two-scale difference equations: I. Existence and global regularity of solutions, SIAM J. Math. Anal. 22 (1991), 1388-1410. MR 92d:39001

5. T. B. Dinsenbacher and D. P. Hardin, Nonhomogeneous refinement equations, Wavelets, Multiwavelets, and their Applications, A. Aldroubi and E. Lin (eds.), AMS Contemporary Mathematics Series 216, 1998, 117-128. MR 99a:39055

6. T. B. Dinsenbacher and D. P. Hardin, Multivariate nonhomogeneous refinement equations, J. Fourier Anal. Appl., to appear.

7. T. N. T. Goodman, R. Q. Jia, and C. A. Micchelli, On the spectral radius of a bi-infinite periodic and slanted matrix, Southeast Asian Bull. Math. 22 (1998), 115-134. MR 2000b:15009

8. B. Han and R. Q. Jia, Multivariate refinement equations and convergence of subdivision schemes, SIAM J. Math. Anal. 29 (1998), 1177-1199. MR 99f:41018

9. C. Heil and D. Colella, Matrix refinement equations: existence and uniqueness, J. Fourier Anal. Appl. 2 (1996), 363-377. MR 97k:39021

10. R. Horn and C. Johnson, Topics in Matrix Analysis, Cambridge University Press, 1991. MR 92e:15003

11. R. Q. Jia, Subdivision schemes in $L_{p}$ spaces, Advances in Comp. Math. 3 (1995), 309-341. MR 96d:65028

12. R. Q. Jia, Shift-invariant spaces on the real line, Proc. Amer. Math. Soc. 125 (1997), 785-793. MR 97e: 41039

13. R. Q. Jia, Partition of unity and density: a counterexample, Constr. Approx. 13 (1997), 251-260. MR 98c:41011 
14. R. Q. Jia, Convergence of vector subdivision schemes and construction of multiple biorthogonal wavelets, Advances in Wavelets, K. S. Lau (ed.), Springer-Verlag, Singapore, 1998, 199227. CMP 99:13

15. R. Q. Jia, Q. T. Jiang, and Z. W. Shen, Distributional solutions of nonhomogeneous discrete and continuous refinement equations, SIAM J. Math. Anal., to appear.

16. R. Q. Jia, S. D. Riemenschneider, and D. X. Zhou, Vector subdivision schemes and multiple wavelets, Math. Comp. 67 (1998), 1533-1563. MR 99d:42062

17. Q. T. Jiang, On the regularity of matrix refinable functions, SIAM J. Math. Anal. 29 (1998), 1157-1176. MR 99d:42063

18. Q. T. Jiang, Multivariate matrix refinable functions with arbitrary matrix dilation, Trans. Amer. Math. Soc. 351 (1999), 2407-2438. MR 99i:42047

19. Q. T. Jiang and Z. W. Shen, On the existence and weak stability of matrix refinable functions, Constr. Approx. 15 (1999), 337-353. MR 2000c:42034

20. W. Lawton, S. L. Lee and Z. W. Shen, Convergence of multidimensional cascade algorithms, Numer. Math. 78 (1998), 427-438. MR 98k:41027

21. W. R. Madych, Finite orthogonal transforms and multiresolution analyses on intervals, J. Fourier Anal. Appl. 3 (1997), 257-294. MR 99c:42067

22. Z. W. Shen, Refinable function vectors, SIAM J. Math. Anal. 29 (1998), 235-250. MR 99d:41038

23. G. Strang, Eigenvalues of $(\downarrow 2) H$ and convergence of the cascade algorithm, IEEE Trans. Signal Processing 44 (1996), 233-238.

24. G. Strang and T. Nguyen, Wavelets and Filter Banks, Wellesley-Cambridge Press, Wellesley, U.S.A., 1996. MR 98b:94003

25. G. Strang and D. X. Zhou, Inhomogeneous refinement equations, J. Fourier Anal. Appl. 4 (1998), 733-747. MR 98m:42056

26. Q. Y.Sun, Nonhomogeneous refinement equations: Existence, regularity and biorthogonality, preprint, 1998.

27. D. X. Zhou, Existence of multiple refinable distributions, Michigan Math. J. 44 (1997), 317329. MR 99a:41021

Department of Mathematical Sciences, University of Alberta, Edmonton, Canada T6G 2G1

E-mail address: jia@xihu.math.ualberta.ca

Department of Mathematics, National University of Singapore, Singapore 119260

E-mail address: qjiang@haar.math.nus.edu.sg

Current address: Department of Mathematical Sciences, University of Alberta, Edmonton, Alberta, Canada T6G 2G1

Department of Mathematics, National University of Singapore, Singapore 119260

E-mail address: matzuows@leonis.nus.edu.sg 